\title{
AN ACCOUNT OF THE THEORY OF CRYSTALLOGRAPHIC GROUPS ${ }^{1}$
}

\section{LOUIS AUSLANDER}

Introduction. L. Bieberbach in two fundamental papers [2], [3] established the fundamental theorems for the crystallographic groups or Raumgruppen. We propose in this paper to give an almost completely self-contained account of these fundamental facts. We will use only the elementary theory of groups, matrices and polynomials from algebra, the basic geometry of euclidean space and the most elementary topological considerations. At one point we will need the exponential mapping for Lie matrix groups for which various elementary accounts are available.

I would like to thank M. Rosenlicht and P. Fong for useful conversations.

1. Definition of crystallographic groups. Let $E^{n}$ denote the $n$ dimensional euclidean space and let $R(n)$ denote the group of rigid motions of $E^{n}$. Then let 0 be a point in $E^{n}$. The subgroup of $R(n)$ leaving 0 fixed is called the orthogonal group and we will denote it by $O(n)$. Let $R^{n}$ be the subgroup of $R(n)$ consisting of pure translations. Then there are two facts which should be recalled: First, $R^{n}$ may be identified with $E^{n}$ under the map $r \in R^{n}$ goes into $r(0)$. Secondly, $R^{n}$ is a normal subgroup of $R(n), O(n) \cap R^{n}$ is empty and every element of $R(n)$ can be uniquely represented in the form $g t$, where $g \in O(n)$ and $t \in R^{n}$. These last three conditions are abbreviated by writing $R(n)=O(n) \cdot R^{n}$.

A subgroup $\Gamma \subset R(n)$ is called a crystallographic group if the following two conditions are satisfied:

1. If $\gamma_{1}, \cdots, \gamma_{n}, \cdots$ is a sequence of elements from $\Gamma$ and $x \in E^{n}$, then the sequence $\gamma_{i} x, i=1,2, \cdots$, is Cauchy if and only if there exists $N>0$ such that $\gamma_{i}=\gamma_{N}$ for all $i>N$.

2. There exists a compact subset of $E^{n}$, say $F$, such that for every $x \in E^{n}$ there exists $\gamma \in \Gamma$ with the property that $\gamma(x) \in F$.

These two conditions are slightly awkward to work with. The following theorem gives a more convenient formulation of the crystallographic groups.

Received by the editors February 17, 1964.

1 During part of the time this paper was being prepared, the author received support from the U. S. Naval Research Laboratory. 
Theorem 1. A necessary and sufficient condition for a subgroup of $R(n)$ to be a crystallographic group is that $\Gamma$ is a discrete subset of $R(n)$ and $R(n) / \Gamma$ is compact in the quotient topology.

Proof. We will prove this theorem by means of the following two propositions.

(A) A necessary and sufficient condition for a subgroup $\Gamma$ of $R(n)$ to act on $E^{n}$ without accumulation points (i.e., so as to satisfy condition (1) of the definition of crystallographic groups) is that $\Gamma$ be a discrete subset of $R(n)$.

For let $\Gamma$ be a discrete subgroup of $R(n)$ and assume there exist $x_{0} \in E^{n}$ and $\gamma_{i} \in \Gamma, i=1, \cdots$, such that $\gamma_{i}\left(x_{0}\right)$ is Cauchy. Further, let $t_{0} \in R^{n}$ be such that $t_{0}(0)=x_{0}$. Then consider $\gamma_{i} t_{0} \in R(n)$. Now $\gamma_{i}=\left(g_{i}, t_{i}\right)$ and

$$
\boldsymbol{\gamma}_{i} t_{0}=\left(g_{i}, \text { ad }\left(g_{i}\right) t_{0}+t_{i}\right) .^{2}
$$

Since $\gamma_{i}\left(x_{0}\right)$ is Cauchy and is exactly $\left(\operatorname{ad}\left(g_{i}\right) t_{0}+t_{i}\right)(0)$, we have that $\operatorname{ad}\left(g_{i}\right) t_{0}+t_{i}$ is a Cauchy sequence in $R^{n}$. Since $O(n)$ is compact, we can find a subsequence of the $g_{i}$ which is Cauchy. Hence the sequence $\gamma_{i} t_{0}$ is Cauchy. But $R(n) \rightarrow R(n)$ obtained by right multiplication by $t_{0}$ is a homeomorphism and, hence, $\gamma_{i}$ must be Cauchy. Hence it must be trivial from some point on and we have proven our first assertion.

Let $\Gamma \subset R(n)$ operate without accumulation points on $E^{n}$. Assume $\gamma_{i} \in \Gamma$ is a Cauchy sequence and $\gamma_{i}=\left(g_{i}, t_{i}\right)$. We must have, since $R(n)$ is topologically $O(n) \times R^{n}$, that $g_{i}$ and $t_{i}$ are both Cauchy sequences. Hence $t_{i}(0)=\gamma_{i}(0)$ must be Cauchy. This proves (A).

(B) A necessary and sufficient condition for a subgroup $\Gamma$ of $R(n)$ to have a compact fundamental domain (i.e., satisfy (2) in the definition of crystallographic group) is that $R(n) / \Gamma$ be compact.

Proof. It is trivial to verify that $\Gamma$ has a compact fundamental domain is equivalent to $E^{n} / \Gamma$, in the quotient topology, shall be compact. Now there is a well-defined continuous mapping of $R(n) / \Gamma$ into $E^{n} / \Gamma$ obtained by identifying two points of $R(n)$ that differ by an element of $O(n)$ acting to the left. Hence, if $R(n) / \Gamma$ is compact, $E^{n} / \Gamma$ is compact.

But $R(n) / \Gamma$ is compact if there exists $F^{*} C R(n)$ such that $F^{*}$ is compact and every element of $R(n)$ differs from an element of $F^{*}$ by a multiple of $\Gamma$. Clearly, we may choose $F^{*}=O(n) \times F$ where $F$ is compact subset of $R^{n}$. Assuming that $\Gamma$ acts with compact funda-

We are using homogeneous coordinates and the multiplication is given by $\left(g_{1}, t_{1}\right)\left(g_{2}, t_{2}\right)=\left(g_{1} g_{2}, \operatorname{ad}\left(g_{1}\right) t_{2}+t_{1}\right)$. 
mental domain on $E^{n}$ gives an $F$ in $E^{n}$ which may be identified with $F$ in $R^{n}$ by the standard identification of $R^{n}$ and $E^{n}$. The assertion is then trivially verified.

2. Neighborhoods of the identity in $O(n)$. In this section we will establish certain elementary properties of the orthogonal group $O(n)$. Let $e$ always denote the identity element of $O(n)$. Some of this material has already appeared in print in [1] and is included for completeness of exposition.

LEMMA 1. Let $O(n)$ denote the orthogonal group and let $\gamma, \eta \in O(n)$. Then there exists a neighborhood of the identity $U(e)$ such that if $\gamma, \eta \in U(e)$ and $\gamma \eta \neq \eta \gamma$, then $\gamma$ will not commute with $\gamma \eta \gamma^{-1} \eta^{-1}=(\gamma, \eta)$.

Proof. Let us assume that $\gamma$ and $(\gamma, \eta)$ commute. Then $\gamma$ and $\eta \gamma^{-1} \eta^{-1}$ commute. Hence $\eta$ can be represented as a permutation of the invariant spaces of $\gamma^{-1}$ or $\gamma$ amongst themselves followed by a mapping of these spaces onto themselves. Hence, if $\eta$ is sufficiently close to the identity, $\eta$ can only map these invariant spaces on to themselves. Hence $\eta$ and $\boldsymbol{\gamma}$ commute. This proves our lemma.

We will now state a general fact giving a general proof. This is the only fact from Lie-group theory we will use and if the reader is unfamiliar with it he can take it on faith or read it in [4, Chapter 2].

LEMMA 2. Let $G$ be a connected Lie group. Then there exists a neighborhood $W(S)$ of the identity in $G$ such that for any $g_{1}, g_{2} \in W(S)$, $\left(g_{1}, g_{2}\right) \in W(S)$ and the sequence

$$
\left(g_{1}\left(g_{1}, g_{2}\right)\right),\left(g_{1}\left(g_{1}\left(g_{1}, g_{2}\right)\right)\right), \ldots
$$

converges to the identity.

Proof. Choose a canonical coordinate system about the identity in $G$. Then the coordinates of $\left(g_{1}, g_{2}\right)$ can be expressed as a power series in the coordinates of $g_{1}$ and $g_{2}$ with quadratic leading term. This proves our assertion.

LEMMA 3. There exist arbitrarily small neighborhoods $U_{\alpha}$ of the identity in $O(n)$ such that, for all $g \in O(n), g U_{\alpha} g^{-1}=U_{\alpha}$.

Proof. Note merely that the set of elements of $O(n)$ whose eigenvalues $\xi$ satisfy an inequality $|\xi-1|<\epsilon$ is a neighborhood of the identity in $O(n)$.

Definition. A neighborhood of the identity in $O(n)$ satisfying the conclusions of Lemmas 1, 2 and 3 will be called a stable neighborhood of the identity. 


\section{Lemmas on crystallographic groups.}

LEMma 4. Let $\Gamma$ be a crystallographic group and let $x \in E^{n}$. Then the set $\{\gamma(x)\}$ for $\gamma \in \Gamma$ cannot lie in a linear space of dimension less than $n$.

Proof. Assume the lemma is false and that $x_{0} \in E^{n}$ exists such that $\left\{\gamma\left(x_{0}\right)\right\}$ lies in $W$, a proper linear subspace of $E^{n}$. By a new choice of origin in $E^{n}$ we may assume $O(n)$ leaves $x_{0}$ fixed and then $\gamma \in \Gamma$, $\gamma=(g(\gamma), t(\gamma))$ must have $t(\gamma) \in W$.

Since $\Gamma$ is a group, $g(W)=W$ for all $g=g(\gamma)$. Let $W^{\perp}$ be the orthogonal complement of $W$. Then, clearly, since points in $W^{\perp}$ at a distance $d$ from the origin stay at a distance $d, \Gamma$ cannot have a compact fundamental domain. This proves our assertion.

LEMMa 5. Let $\Gamma$ be an abelian crystallographic group; then $\Gamma$ contains only pure translations.

Proof. Let $\gamma_{0} \in \Gamma$ and let $\gamma_{0}=\left(g\left(\gamma_{0}\right), t_{0}\right)$, where $g\left(\gamma_{0}\right) \neq e$. Then we can always choose an origin and a coordinate system in $R^{n}$ such that, using homogeneous coordinates,

$$
\gamma_{0}=\left(\begin{array}{lll}
I & 0 & t_{0} \\
0 & \delta & 0 \\
0 & 0 & 1
\end{array}\right)
$$

where $I$ is the $(r \times r)$ identity matrix, $(\delta-I)$ is a nonsingular $s \times s$ matrix, $t_{0}$ is a $(1 \times r)$ matrix and 1 is a $1 \times 1$ matrix with 1 as an entry and $s+r=n$. Then, by Lemma 4 , there exist $\gamma \in \Gamma$ such that

$$
\gamma=\left(\begin{array}{llr}
A & 0 & t_{1} \\
0 & B & t_{2} \\
0 & 0 & 1
\end{array}\right)
$$

where $A$ is $(r \times r), B$ is $(s \times s), t_{1}$ is $1 \times r$, and $t_{2}$ is a $(1 \times s)$ nontrivial matrix. Then, since $\Gamma$ is abelian, $\gamma_{1} \gamma_{0} \gamma_{1}^{-1}=\gamma_{0}$ and this implies that $(\delta-I) t_{2}=0$. Since $(\delta-I)$ is nonsingular, this is impossible as $t_{2} \neq 0$. This proves our assertion.

\section{Main theorems.}

Proposition 1. Let $\Gamma$ be a discrete subgroup of $R(n)$ and let $\psi$ denote the homomorphism of $R(n)$ onto $O(n)$ with kernel $R^{n}$. Then the identity component of the closure of $\psi(\Gamma)$ in $O(n)$ is abelian. 
Proof. Let $U$ be a stable neighborhood of the identity in $O(n)$ with the further property that for all $g \in U$ all the matrix values of $g$ satisfy $\left|\xi_{i j}-1\right|<1 / 10$. In some orthonormal basis choose $\gamma_{1}, \gamma_{2} \in \Gamma$ with the property that $\psi\left(\gamma_{i}\right) \in U, i=1,2$. Then $\gamma_{i}=\psi\left(\gamma_{i}\right) t\left(\gamma_{i}\right)$, which we will abbreviate $x_{i} y_{i}$.

Then

$$
\left(\gamma_{1}, \gamma_{2}\right)=\left(x_{1}, x_{2}\right) \operatorname{ad}\left(x_{1}^{-1} x_{2}^{-1}\right)\left[\left(\operatorname{ad}\left(x_{2}\right)-I\right) y_{1}+\left(I-\operatorname{ad}\left(x_{1}\right)\right) y_{2}\right],
$$

where $\operatorname{ad}(x)$ is the automorphism of $R^{n}$ induced by $x^{-1} R^{n} x$. We then form the sequence $\gamma_{1},\left(\gamma_{1}, \gamma_{2}\right),\left(\gamma_{1},\left(\gamma_{1} \gamma_{2}\right)\right),\left(\gamma_{1},\left(\gamma_{1},\left(\gamma_{1}, \gamma_{2}\right)\right)\right), \ldots$ By our construction, the coefficients in $O(n)$ and $R^{n}$ of this. sequence are easily seen to be bounded. But by Lemma 1, this series can never be the identity, and, by Lemma 2 , it can never become trivial at any point not the identity. Hence, since $\Gamma$ is discrete, the identity component of the closure of $\psi(\Gamma)$ is abelian.

Bieberbach Theorem 1. Let $\Gamma$ be a crystallographic group; then $\Gamma$ satisfies the following three conditions:

1. $\Gamma \cap R^{n}$ is a vector space basis for $R^{n}$ as a real vector space.

2. $\Gamma / \Gamma \cap R^{n}=F(\Gamma)$ is a finite group.

3. $F(\Gamma)$ has all integer entries with respect to any basis of $R^{n}$ determined by the generators of $\Gamma \cap R^{n}$.

Proof. Assume first that $\Gamma \cap R^{n}$ is trivial. Then $\psi(\Gamma)$ is an isomorphism of $\Gamma$ into $O(n)$ and we will let $I_{0}(\psi(\Gamma))$ denote the identity component of the closure of $\psi(\Gamma)$. Since $O(n)$ is compact, the closure of $\psi(\Gamma)$ can have only a finite number of components. Hence, since $I_{0}(\psi(\Gamma))$ is abelian, $\Gamma$ contains a subgroup $\Gamma_{1}$ of finite index which is abelian. But then $\Gamma_{1}$, being of finite index in $\Gamma$, is also a crystallographic group. Hence, by Lemma $5, \Gamma_{1}$ consists of pure translations. Thus we see that $\Gamma \cap R^{n}$ is nonempty.

Let $W \subset R^{n}$ be the subspace of $R^{n}$ spanned by the pure translations of $\Gamma$, i.e., by $\Gamma \cap R^{n}$. Then, clearly, if $R(n)=O(n) \cdot R^{n}$ again and $\gamma \in \Gamma$ is given by $(g(\gamma), t(\gamma)), g(\gamma) \in O(n), t(\gamma) \in R^{n}$, then $g(\gamma)$ leaves $W$ invariant since $\Gamma \cap R^{n}$ is normal in $\gamma$. Note further that $\{g(\gamma)\} \mid W$ all $\gamma \in \Gamma$ is a finite group, for otherwise it would contain elements arbitrarily close to the identity which would, under inner automorphism with a basis of $\Gamma \cap R^{n}$, force $\Gamma$ to be nondiscrete. From this we see that $\Gamma$ induces an action on $R^{n} / W$ which is that of a crystallographic group with no pure translations. By the first part, this implies the dimension of $R^{n} / W$ is zero.

This discussion verifies part one and part two of the Bieberbach Theorem, part three follows trivially from parts one and two. 
JORDAN THEOREM. There exists a positive function of $n, n \geqq 0, f(n)$, such that, for every finite group $F \subset O(n)$, there exists an abelian normal subgroup $A(F)$ such that the order of $F / A(F)$ is less than $f(n)$.

Proof. Let $U$ be a stable neighborhood of the identity as defined in §2. Let $A(F)$ be the subgroup of $F$ generated by $F \cap U$. The definition of $U$ insures that $A(F)$ is normal and abelian. Now assume $O(n)$ has Haar measure with total measure 1. Let the measure of $U>1 / m$, $m$ an integer. Then it is easily seen that the order of $F / A(F)$ must be less than $m$.

TheOREM 2. Let $F_{\alpha}, \alpha=1, \cdots, k$, be the set of subgroups of $O(n)$ which can be expressed as integer matrices with determinant \pm 1 in $\mathrm{GL}(n, R)$. Then $k$ is a finite cardinal.

Proof. A subgroup of a group satisfying our hypothesis again satisfies our hypothesis. Let $A_{\alpha}$ be the normal subgroup of $F_{\alpha}$ described in the Jordan Theorem. Since the order of $F_{\alpha} / A_{\alpha}$ is bounded, there exist only a finite number of distinct groups of the form $F_{\alpha} / A_{\alpha}$, $\alpha=1, \cdots, k$. If we can show there exist only a finite number of $A_{\alpha}$, we will have proven our assertion as the group extensions must then also be finite. Now $A_{\alpha}$ is the abelian semisimple group. We will show that there are only a finite number of elements of $O(n)$ which can be in $A_{\alpha}$ for all $\alpha$. Hence $A_{\alpha}$ must consist of a finite collection of groups. First note that $n$ times the distinct characteristic polynomials is greater than the number of distinct elements of $O(n)$ in $A_{\alpha}$. But since all roots have absolute value one and the coefficients of the characteristic polynomials are the elementary symmetric functions, they can take on at most a finite number of values. This completes the proof.

CoROllary. Let $\Gamma$ be a crystallographic group and $R^{n}$ the group of pure translations. Then, for each $n$, there exist only a finite number of groups $\Gamma / \Gamma \cap R^{n}$.

BIEBERBACH TheOREM 2. For each $n$, there exist only a finite number of crystallographic groups.

Proof. We have seen that $\Gamma$ satisfies an exact diagram

$$
1 \rightarrow Z^{n} \rightarrow \Gamma \rightarrow F \rightarrow 1,
$$

where $Z^{n}$ is $n$ copies of the integers and $F$ can range over a finite collection of groups. It is well known that for each finite group $F$ there are only a finite number of nonisomorphic groups satisfying the above diagram. This completes our argument. 


\section{REFERENCES}

1. L. Auslander, Bierberbach's theorem on space groups and discrete uniform subgroups of Lie groups. II, Amer. J. Math. 83 (1961), 276-280.

2. L. Bieberbach, Über die Bewegungsgruppen der Euklidischen Raüme. I, Math. Ann. 70 (1911), 297-336.

3. - Über die Bewegungsgruppen der Euklidischen Raume. II, Math. Ann. 72 (1912), 400-412.

4. S. Helgason, Differential geometry and symmetric spaces, Academic Press, New York, 1962.

Purdue University and

University of California, Berkeley

\section{EXTENSION OF NORMAL FAMILIES OF HOLOMORPHIC FUNCTIONS}

\section{THEODORE J. BARTH ${ }^{1}$}

Let $X$ be a Stein manifold, and let $A$ be an analytic subset of $X$ $\mathrm{A}$ well-known application of Cartan's Theorem $\mathrm{B}$ [2, Théorème 3 p. 52] states that each holomorphic function on $A$ is the restriction of a holomorphic function on $X$. This paper presents a generalization of this application, namely that each normal family of holomorphic functions on $A$ is the restriction of a normal family of holomorphic functions on $X$.

1. Let $X$ be a topological space which is $\sigma$-compact, i.e., the union of a countable family of compact sets. Let $K(X)$ denote the set of all compact subsets of $X$. For $K \in K(X)$ and $f: X \rightarrow C$ define $\|f\|_{K}$ $=\sup \{|f(x)| \mid x \in K\}$. Define

$$
B(X)=\left\{f \mid f: X \rightarrow C,\|f\|_{K}<\infty \text { for all } K \in K(X)\right\} .
$$

Clearly $B(X)$ is a complex vector space, and $\left\{\|\|_{K} \mid K \in K(X)\right\}$ is a family of pseudonorms on $B(X)$ which then becomes a locally convex vector space. Since $X$ is $\sigma$-compact, $B(X)$ is metrizable, and it is readily checked to be a Fréchet space.

Definition. Let $V$ be a vector subspace of $B(X)$. We say that a set $F \subset V$ is normal with respect to $V$ iff every sequence in $F$ has a subsequence which converges in $V$.

Received by the editors October 29, 1964.

1 This research supported in part by the National Science Foundation while the author held a Graduate Fellowship at the University of Notre Dame. 